

\title{
A Molecular Based Dynamic Model for Viscoelastic Responses of Rubber in Tensile Deformations*
}

November 8, 2000

\author{
H.T. Banks \\ Center for Research in Scientific Computation \\ North Carolina State University \\ Raleigh, NC 27695-8205 \\ N.G. Medhin \\ Department of Mathematics \& \\ Center for Theoretical Studies of Physical Systems \\ Clark Atlanta University \\ Atlanta, GA 30314
}

${ }^{*}$ This research was supported in part (H.T. Banks) by the Air Force Office of Scientific Research under grant AFOSR F49620-98-1-0180 and in part (N.G. Medhin) by the National Science Foundation under cooperative agreement \# HRD-9632844. 


\begin{abstract}
Dynamic equations are developed for rubber viscoelasticity based upon a stick-slip continuum molecular based model. The model considered is a continuum simulation of a tube reptation model in which a chemically cross-linked (CC) system of molecules act as a constraint box per unit volume for a physically constrained (PC) system of molecules. The CC-system carries along the $\mathrm{PC}$-system during instantaneous step deformations. The subsequent relaxation of the $\mathrm{PC}$-system is determined by the history of the CC-system and thermodynamic considerations. At the same time the $\mathrm{PC}$-system deformation acts as an internal variable affecting the relaxation of the CC-system. Modeling of this relaxation process and subsequent employment of energy density functions constitutes one basis to model viscoelastic effects in rubber deformations.
\end{abstract}




\section{Introduction}

Various molecular and phenomenological models have been developed to model both small and large deformations in rubber. For a recent review, see the survey by Johnson $[\mathrm{J}]$. To provide a more accurate and realistic model of the deformation and relaxation process in rubber molecular theory $[\mathrm{S}],[\mathrm{T}]$ and continuum mechanics $[\mathrm{O}]$, it is useful to integrate various approaches.

In this paper we use the molecular models of Doi/Edwards [DE] and Johnson/Stacer [JS] to derive a class of nonlinear distributed parameter systems (partial differential equations) with internal strain dynamics that include the pseudo-phenomenological models of [BPP], [BPPGY]. These latter models have provided good agreement with both quasi-static and dynamic data for rubber in uniaxial tension and in shear.

The dynamic model for rubber viscoelasticity presented here is based on the continuum simulation [JS] of a tube reptation model considered in [DE]. In [DE] step-strain relaxation of polymers is modeled with constraint (stickslip) theory in which $\mathrm{PC}$-molecules deform with CC-molecules during a large step-strain. Then the $\mathrm{PC}$-molecules contract and creep to return to a low energy and higher entropy state. As a result, the total energy density at a constraint strain dissipates in time and a viscoelastic theory results. These models are based on cross-linking rubber network theories for rubbers and other polymers [S], [T].

A continuum molecular model of rubber viscoelasticity is proposed in [JS] and entails consideration of the chemically cross-linked molecules as providing cells or boxes with entrapped molecular segments. The model involves placing a unit cell or box at each point of the rubber continuum and deriving subsequent equations for the associated principal stretches.

\section{The Continuum Model of Johnson and Stacer}

In this section and the next we summarize the arguments of Johnson and Stacer in [JS]. Let the CC constraint tube have length $L(t)$ and the entrapped PC-molecule have length $\ell(t)$. The model under consideration assumes that the length of the $\mathrm{CC}$ tube is approximated as a step function of time and $\ell(0)=L(0)$. Suppose an instantaneous tensile step-strain deformation at 
time of the $\mathrm{CC}$-system results in the $\mathrm{PC}$ molecule having length $\ell^{*}$, and a model of the $\mathrm{PC}$-molecule returning to its original contour length is given for $t>0$ by

$$
\ell(t)=\ell(0)+\left[\ell^{*}-\ell(0)\right] e^{-t / \tau} .
$$

If the instantaneous stretch at a time $t_{0}$ for the CC-tube is $\Delta L_{0}$, and that for the $\mathrm{PC}$ molecule is $\Delta \ell_{0}$, and if we relate the stretches in the form $\frac{\Delta \ell_{0}}{\Delta L_{0}}=\frac{\ell_{0}}{L_{0}}$, we find the relation

$$
\Delta \ell_{0}=\left(\frac{\ell_{0}}{L_{0}}\right) \Delta L_{0} .
$$

Then, in the time interval $t_{0}<t<t_{1}$ we have

$$
\ell(t)=\ell_{0}+\frac{\ell_{0}}{L_{0}} \Delta L_{0} e^{-\left(t-t_{0}\right) / \tau}
$$

where $\tau$ is the relaxation time for the slip motion. Next we suppose that the CC-box is subjected to further instantaneous tensile step-deformations of magnitudes $\Delta L_{i}$ at times $t_{i}, i=1,2, \ldots$. Setting $t=t_{1}$, we find

$$
\begin{gathered}
\ell\left(t_{1}\right)=\ell_{0}+\frac{\ell_{0}}{L_{0}} \Delta L_{0} e^{-\left(t_{1}-t_{0}\right) / \tau}, \\
L_{1}=L_{0}+\Delta L_{0} .
\end{gathered}
$$

For $t$ in the time interval $\left(t_{1}, t_{2}\right)$, we have

$$
\begin{aligned}
\ell(t) & =\ell_{0}+\left[\left(\ell_{1}+\Delta \ell_{1}\right)-\ell_{0}\right] e^{-\left(t-t_{1}\right) / \tau} \\
& =\ell_{0}+\left[\frac{\ell_{0}}{L_{0}} \Delta L_{0} e^{-\left(t_{1}-t_{0}\right) / \tau}+\frac{\ell_{1}}{L_{1}} \Delta L_{1}\right] e^{-\left(t-t_{1}\right) / \tau} \\
& =\ell_{0}+\frac{\ell_{0}}{L_{0}} \Delta L_{0} e^{-\left(t-t_{0}\right) / \tau}+\frac{\ell_{1}}{L_{1}} \Delta L_{1} e^{-\left(t-t_{1}\right) / \tau} .
\end{aligned}
$$

Similarly for $t_{2}<t<t_{3}$ we have

$$
\ell(t)=\ell_{0}+\frac{\ell_{0}}{L_{0}} \Delta L_{0} e^{-\left(t-t_{0}\right) / \tau}+\frac{\ell_{1}}{L_{1}} \Delta L_{1} e^{-\left(t-t_{1}\right) / \tau}+\frac{\ell_{2}}{L_{2}} \Delta L_{2} e^{-\left(t-t_{2}\right) / \tau} .
$$

Passing to the limit as $\Delta t_{i}=t_{i}-t_{i-1} \rightarrow 0$, we are thus led to the formula

$$
\ell(t)=\ell_{0}+\int_{0}^{t} \frac{\ell(s)}{L(s)} \frac{d L(s)}{d s} e^{-(t-s) / \tau} d s
$$


for the length of the $\mathrm{PC}$-molecule at any time $t$ in terms of the previous lengths $l(s), L(s)$ and the rate of change $\frac{d L}{d s}(s)$. In differential form this becomes

$$
\frac{d l}{d t}=\frac{l_{0}}{\tau}-\left(\frac{1}{\tau}-\frac{1}{L} \frac{d L}{d t}\right) l
$$

\section{$3 \quad$ Strain Energy Density}

To use the stick-slip model in continuum simulation of the reptation model of rubber elasticity one considers a network of cells or boxes in the rubber continuum with sides $\lambda_{1}, \lambda_{2}$, and $\lambda_{3}$. The CC-box will have positive strain energy density $W_{c c}\left(\lambda_{1 c}, \lambda_{2 c}, \lambda_{3 c}\right)$ for all stretches except when $\lambda_{1 c}=\lambda_{2 c}=$ $\lambda_{3 c}=1$. Next, a box for the PC-system with sides parallel to those of the CC-box is defined by sides $\lambda_{1 p}, \lambda_{2 p}, \lambda_{3 p}$ along with an energy density $W_{p c}\left(\lambda_{1 p}, \lambda_{2 p}, \lambda_{3 p}\right)$.

Stresses are calculated from the strain energy density [VL] by determining how the energy density function changes with respect to changes in the stretches or displacements. In the model considered, the strain energy density of the rubber continuum is assumed to have the form

$$
\begin{aligned}
W & =W_{c c}+W_{p c} \\
& =W_{c c}\left(\lambda_{1 c}, \lambda_{2 c}, \lambda_{3 c}\right)+W_{p c}\left(\lambda_{1 p}, \lambda_{2 p}, \lambda_{3 p}\right) .
\end{aligned}
$$

To find the stresses at a generic point of the rubber continuum one must determine how $W$ changes with respect to stretches of the CC-system. The stretches of the $\mathrm{PC}$-boxes are then treated as internal variables depending on the stretches of the CC-system.

The Cauchy stress in the principal direction $e_{j}$, where $e_{j}$ is a unit vector in the $x_{j}$ direction, is given by

$$
\tau_{j}=\lambda_{j c} \frac{\partial W}{\partial \lambda_{j c}}-P
$$

where $P$ is the hydrostatic stress. If we consider (4) with the $\lambda_{i p}$ as internal variables depending on the $\lambda_{i c}$, (5) becomes 


$$
\tau_{j}=\lambda_{j c} \frac{\partial W_{c c}}{\partial \lambda_{j c}}+\lambda_{j c} \sum_{i=1}^{3} \frac{\partial W_{p c}}{\partial \lambda_{i p}} \frac{\partial \lambda_{i p}}{\partial \lambda_{j c}}-P .
$$

In obtaining (3) we have related the instantaneous tensile step deformations $\Delta \ell_{k}, \Delta L_{k}$ at $t=t_{k}$ according to the formula

$$
\frac{\Delta \ell_{k}}{\Delta L_{k}}=\frac{\ell_{k}}{L_{k}}
$$

Using this observation in the analogy of $\Delta l_{k}, \Delta L_{k}$ with principal stretches $\lambda_{i p}, \lambda_{i c}$, respectively, we may write

$$
\frac{\partial \lambda_{j p}}{\partial \lambda_{i c}}=\frac{\lambda_{j p}}{\lambda_{i c}} \delta_{j i}
$$

In the case of tensile deformations, choosing $j=1$ for the direction of loading and using (7) and (4) in (6), we have

$$
\tau_{2}=\tau_{3}=0=\lambda_{2 c} \frac{\partial W_{c c}}{\partial \lambda_{2 c}}+\lambda_{2 p} \frac{\partial W_{p c}}{\lambda_{2 p}}-P .
$$

With the hydrostatic stress determined from (8), the tensile Cauchy stress is thus given by

$$
\tau_{1}=\left(\lambda_{1 c} \frac{\partial W_{c c}}{\partial \lambda_{1 c}}-\lambda_{2 c} \frac{\partial W_{c c}}{\partial \lambda_{2 c}}\right)+\left(\lambda_{1 p} \frac{\partial W_{p c}}{\partial \lambda_{1 p}}-\lambda_{2 p} \frac{\partial W_{p c}}{\partial \lambda_{2 p}}\right)
$$

\section{A Dynamic Continuum Model}

To demonstrate how the box model detailed above can be applied to the analysis of rubber undergoing large dynamic tensile strains, we consider the strain energy function suggested by Johnson and Stacer (based on experimental data of Young and Danik - see [JQYD]) given by

$$
\begin{array}{r}
W_{c c}=105.0\left(I_{1}-3\right)+103.0\left(I_{2}-3\right), \\
W_{p c}=169.0\left(I_{1}-3\right)+0.0138\left(I_{1}-3\right)^{2}+7.89\left(I_{2}-3\right)^{3},
\end{array}
$$

where $I_{1}=\lambda_{1 c}^{2}+\lambda_{2 c}^{2}+\lambda_{3 c}^{2}$, and $I_{2}=\lambda_{1 c}^{2} \lambda_{2 c}^{2}+\lambda_{1 c}^{2} \lambda_{3 c}^{2}+\lambda_{2 c}^{2} \lambda_{3 c}^{2}$. 
We also impose the incompressibility condition $\lambda_{1} \lambda_{2} \lambda_{3}=1$ on the principal stretches $\lambda_{c c}=\left(\lambda_{1 c}, \lambda_{2 c}, \lambda_{3 c}\right)$ and $\lambda_{p c}=\left(\lambda_{1 p}, \lambda_{2 p}, \lambda_{3 p}\right)$.

From (9) the engineering stress $\sigma_{1}=\tau_{1} / \lambda_{1 c}$ is given by

$$
\sigma_{1}=\frac{\partial W_{c c}}{\partial \lambda_{1 c}}-\frac{\lambda_{2 c}}{\lambda_{1 c}} \frac{\partial W_{c c}}{\partial \lambda_{2 c}}+\frac{\lambda_{1 p}}{\lambda_{1 c}} \frac{\partial W_{p c}}{\partial \lambda_{1 p}}-\frac{\lambda_{2 p}}{\lambda_{1 c}} \frac{\partial W_{p c}}{\partial \lambda_{2 p}}
$$

where, from (10), we have

$$
\begin{aligned}
\frac{\partial W_{c c}}{\partial \lambda_{1 c}}-\frac{\lambda_{2 c}}{\lambda_{1 c}} \frac{\partial W_{c c}}{\partial \lambda_{2 c}}= & 210 \lambda_{1 c}+206\left(\lambda_{1 c} \lambda_{2 c}^{2}+\lambda_{1 c} \lambda_{3 c}^{2}\right) \\
= & -\frac{\lambda_{2 c}}{\lambda_{1 c}}\left(210 \lambda_{2 c}+206\left(\lambda_{1 c}^{2} \lambda_{2 c}+\lambda_{3 c}^{2} \lambda_{2 c}\right)\right) \\
= & 210 \lambda_{1 c}+206 \lambda_{1 c} \lambda_{2 c}^{2}+206 \lambda_{1 c} \lambda_{3 c}^{2}- \\
& \frac{210 \lambda_{2 c}^{2}}{\lambda_{1 c}}-\frac{206 \lambda_{1 c}^{2} \lambda_{2 c}^{2}}{\lambda_{1 c}}-\frac{206 \lambda_{3 c}^{2} \lambda_{2 c}^{2}}{\lambda_{1 c}} .
\end{aligned}
$$

For the tension in the incompressible CC-system we see that

$$
\lambda_{2 c}=\lambda_{3 c}=\frac{1}{\sqrt{\lambda_{1 c}}}
$$

Then, from (12), we can compute

$$
\begin{aligned}
& \frac{\partial W_{c c}}{\partial \lambda_{1 c}}-\frac{\lambda_{2 c}}{\lambda_{1 c}} \frac{\partial W_{c c}}{\partial \lambda_{2 c}}= \\
& 210 \lambda_{1 c}+206-\frac{210}{\lambda_{1 c}^{2}}-\frac{206}{\lambda_{1 c}^{3}} .
\end{aligned}
$$

Next we deal with the PC-molecules and find

$$
\begin{aligned}
W_{p c}= & 169.0\left(\lambda_{1 p}^{2}+\lambda_{2 p}^{2}+\lambda_{3 p}^{2}-3\right)+0.0318\left(\lambda_{1 p}^{2}+\lambda_{2 p}^{2}+\lambda_{3 p}^{2}-3\right)^{2}+ \\
& 7.89\left(\lambda_{1 p}^{2} \lambda_{2 p}^{2}+\lambda_{1 p}^{2} \lambda_{3 p}^{2}+\lambda_{2 p}^{2} \lambda_{3 p}^{2}-3\right)^{3}
\end{aligned}
$$

Using the relationship

$$
\lambda_{2 p}=\lambda_{3 p}=\frac{1}{\sqrt{\lambda_{1 p}}}
$$


for tension, we obtain from (14)

$$
\begin{aligned}
\frac{\lambda_{1 p}}{\lambda_{1 c}} & \frac{\partial W_{p c}}{\partial \lambda_{1 p}}-\frac{\lambda_{2 p}}{\lambda_{1 c}} \frac{\partial W_{p c}}{\partial \lambda_{2 p}}= \\
& {\left[338.0+0.0552\left(2 \lambda_{1 p}+\frac{1}{\lambda_{1 p}^{2}}-3\right)\right] \frac{1}{\lambda_{1 c}}\left(\lambda_{1 p}^{2}-\frac{1}{\lambda_{1 p}}\right)+\ldots }
\end{aligned}
$$

Letting $u_{c}$ represent the deformation of the CC-box we have

$$
\begin{aligned}
\lambda_{1 c} & =1+\partial_{x} u_{c} \\
\lambda_{1 c}^{-2} & \approx 1-2 \partial_{x} u_{c} \\
\lambda_{1 c}^{-3} & \approx 1-3 \partial_{x} u_{c}
\end{aligned}
$$

while

$$
\lambda_{1 p}=1+\partial_{x} u_{p}
$$

Then

$$
\begin{aligned}
\frac{\partial W_{c c}}{\partial \lambda_{1 c}}-\frac{\lambda_{2 c}}{\lambda_{1 c}} \frac{\partial W_{c c}}{\partial \lambda_{2 c}} \approx & 210\left(1+\partial_{x} u_{c}\right)+206-210\left(1-2 \partial_{x} u_{c}\right) \\
& -206\left(1-3 \partial_{x} u_{c}\right) \\
= & 1248 \partial_{x} u_{c} .
\end{aligned}
$$

Let $u_{p}$ represent the deformation of the PC-box. Then, proceeding as above

$$
\frac{\lambda_{1 p}}{\lambda_{1 c}} \frac{\partial W_{p c}}{\partial \lambda_{1 p}}-\frac{\lambda_{2 p}}{\lambda_{1 p}} \frac{\partial W_{p c}}{\partial \lambda_{2 p}} \approx 1014\left(1-\partial_{x} u_{c}\right) \partial_{x} u_{p} .
$$

Thus, using (11) we obtain the engineering stress approximation

$$
\begin{aligned}
\sigma & \approx 1248 \partial_{x} u_{c}+1014\left(1-\partial_{x} u_{c}\right) \partial_{x} u_{p} \\
& =\left(1248-1014 \partial_{x} u_{p}\right) \partial_{x} u_{c}+1014 \partial_{x} u_{p}
\end{aligned}
$$

In (3) taking $\lambda_{1 p}(t)$ for $\ell(t), \lambda_{1 c}(t)$ for $L(t)$ and using $l_{0}=l(0)=\lambda_{1 p}(0)=1$, we find 


$$
\frac{d \lambda_{1 p}}{d t}=\frac{1}{\tau}-\left[\frac{1}{\tau}-\frac{1}{\lambda_{1 c}} \frac{d \lambda_{1 c}}{d t}\right] \lambda_{1 p} .
$$

Using the definitions $\lambda_{1 p}=1+\partial_{x} u_{p}, \quad \lambda_{1 c}=1+\partial_{x} u_{c}$ in (20) we have

$$
\frac{\partial}{\partial t}\left(1+\partial_{x} u_{p}\right)=1 / \tau-\left\{1 / \tau-\left(1+\partial_{x} u_{c}\right)^{-1} \frac{\partial^{2} u_{c}}{\partial t \partial x}\right\}\left(1+\partial_{x} u_{p}\right) .
$$

In terms of the infintesimal strains $\varepsilon_{1}=\partial_{x} u_{p}$ for the $\mathrm{PC}$ system and $\varepsilon=\partial_{x} u_{c}$ for the CC system, we have

$$
\tau \dot{\varepsilon}_{1}+\varepsilon_{1}=\tau \dot{\varepsilon}\left(1+\varepsilon_{1}\right) /(1+\varepsilon) .
$$

If we use the approximation $(1+\varepsilon)^{-1} \approx 1-\varepsilon+\ldots,(22)$ provides a relationship for "internal dynamics"

$$
\tau \dot{\varepsilon}_{1}+\varepsilon_{1}=\tau \dot{\varepsilon}\left(1+\varepsilon_{1}\right)(1-\varepsilon) .
$$

Ignoring the terms $\dot{\varepsilon} \varepsilon_{1} \varepsilon$ in (23), we obtain a further approximation of the internal dynamics given by

$$
\dot{\varepsilon}_{1}+\frac{1}{\tau} \varepsilon_{1}=\dot{\varepsilon}+\dot{\varepsilon}\left[\varepsilon_{1}-\varepsilon\right] .
$$

We remark that in integrated form the equation (24) can be written (using an integration by parts after integration)

$$
\varepsilon_{1}(t)=\varepsilon(t)-\varepsilon(0) e^{-t / \tau}-\frac{1}{\tau} \int_{0}^{t} \varepsilon(s) e^{-(t-s) / \tau} d s+\int_{0}^{t}\left[\varepsilon_{1}(s)-\varepsilon(s)\right] \dot{\varepsilon}(s) e^{-(t-s) / \tau} d s .
$$

In terms of the deformations $\partial_{x} u_{p}, \partial_{x} u_{c}$ this is the same as

$$
\begin{aligned}
\partial_{x} u_{p}= & \partial_{x} u_{c}-\frac{1}{\tau} \int_{0}^{t} \partial_{x} u_{c}(s, x) e^{-(t-s) / \tau} d s \\
& +\int_{0}^{t}\left[\partial_{x} u_{p}(s, x)-\partial_{x} u_{c}(s, x)\right] \frac{\partial^{2} u_{c}(s, x)}{\partial s \partial x} e^{-(t-s) / \tau} d s \\
& -\partial_{x} u_{c}(0, x) e^{-t / \tau} .
\end{aligned}
$$


We next consider the longitudinal vibration of a rubber rod with (undeformed) cross-sectional area $A_{c}$ and mass density $\rho$. Using (19) we write the equation of motion (recall that $S=A_{c} \sigma$ is the engineering stress resultant see $(2.1)$ of $[\mathrm{BPPGY}])$

$$
\rho A_{c} \partial_{t}^{2} u_{c}-\partial_{x}\left[A_{c}\left(1248-1014 \partial_{x} u_{p}\right) \partial_{x} u_{c}\right]-\partial_{x}\left(1014 A_{c} \partial_{x} u_{p}\right)=q,
$$

where $q$ is the applied load (in force/unit length) and this equation must be solved with (26) (or equivalently (22)), or one of its approximations (23) or (24) to provide a defining coupling for $\partial_{x} u_{p}$ and $\partial_{x} u_{c}$.

In summary, the full nonlinear model we have derived for the CC-PC molecular system in tensile deformations for a rod have the form

$$
\begin{gathered}
\rho \partial_{t}^{2} u_{c}-\partial_{x} \sigma\left(\varepsilon, \varepsilon_{1}\right)=q / A_{c} \\
\sigma\left(\varepsilon, \varepsilon_{1}\right)=\left(1248-1014 \varepsilon_{1}\right) \varepsilon+1014 \varepsilon_{1} \\
\tau \dot{\varepsilon}_{1}+\varepsilon_{1}=\tau \dot{\varepsilon}\left(1+\varepsilon_{1}\right) /(1+\varepsilon)
\end{gathered}
$$

with $\varepsilon=\partial_{x} u_{c}, \varepsilon_{1}=\partial_{x} u_{p}$ and $q$ the applied load (in linear force density).

We remark that the system (27), (23) can be viewed as a member of a class of systems similar to those treated in $[\mathrm{ABP}]$ and the methods presented there can be used to guarantee well-posedness (existence and uniqueness) of the corresponding variational forms of this system. The models treated in $[\mathrm{ABP}]$ can be properly viewed as generalizations of $(27),(23)$ that permit nonlinear instantaneous elastic responses as well as other refinements.

\section{Qualitative Analysis}

In this section we turn to a brief discussion of some of the qualitative properties of the model derived in the previous section. We first linearize (29) to obtain

$$
\sigma_{L}(\varepsilon, \dot{\varepsilon})=1248 \varepsilon+1014 \varepsilon_{1} .
$$

We use the linearized version of (30) - essentially (24) in which we ignore the term $\dot{\varepsilon}\left(\varepsilon_{1}-\varepsilon\right)$ - given by 


$$
\dot{\varepsilon}_{1}+\frac{1}{\tau} \varepsilon_{1}=\dot{\varepsilon}
$$

Integrating and using $\varepsilon(0)=\partial_{x} u_{c}(0, x)=0$, we obtain

$$
\varepsilon_{1}(t)=\varepsilon(t)-\frac{1}{\tau} \int_{0}^{t} \varepsilon(s) e^{-(t-s) / \tau} d s .
$$

This results in the linearized system

$$
\rho \partial_{t}^{2} u_{c}-2262 \partial_{x}^{2} u_{c}+\frac{1014}{\tau} \int_{0}^{t} \partial_{x}^{2} u_{c}(s, \cdot) e^{-(t-s) / \tau} d s=q / A_{c} .
$$

Making the change of dependent variable given by

$$
v=\frac{2262}{\rho} u_{c}-\frac{1014}{\rho \tau} \int_{0}^{t} u_{c}(s, x) e^{-(t-s) / \tau} d s,
$$

we obtain

$$
\begin{aligned}
v_{t t}+ & \frac{1014}{2262 \tau} v_{t}-\frac{1248}{2262 \tau^{2}} v-\frac{2262}{\rho} v_{x x} \\
& +\left(\frac{1248}{2262 \tau}\right)^{2} \int_{0}^{t} e^{-\frac{1248}{2262 \tau}(t-s)}\left(v_{t}+\frac{1}{\tau} v\right) d s=f,
\end{aligned}
$$

where

$$
f=\frac{2262}{\rho^{2} A_{c}} q .
$$

Next consider the standard Sturm-Liouville problem resulting from separation of variables in systems such as equation (36). The generic problem is given by

$$
\begin{aligned}
-y^{\prime \prime}-\lambda y & =0 \\
y(a)-h_{0} y^{\prime}(a) & =0, h_{0} \geq 0 \\
y(b)+h_{1} y^{\prime}(b) & =0, h_{1} \geq 0 .
\end{aligned}
$$

For this problem it is known [CL] that there is a sequence of eigenvalues $0<\lambda_{1}<\lambda_{2} \ldots<\lambda_{n}<\ldots \nearrow \infty$ and corresponding complete family of orthonormal eigenfunctions $\varphi_{1}, \varphi_{2}, \ldots, \varphi_{n}, \ldots$ 
From this one is led to consider a solution of (36) in the form

$$
v(t, x)=\sum_{n=1}^{\infty} v_{n}(t) \varphi_{n}(x) .
$$

Also expanding the forcing function

$$
f(t, x)=\sum_{n=1}^{\infty} f_{n}(t) \varphi_{n}(x)
$$

we formally obtain from (36) the equations

$$
\begin{aligned}
\ddot{v}_{n}(t) & +\frac{1014}{2262 \tau} \dot{v}_{n}(t)+\left(\frac{2262 \lambda_{n}}{\rho}-\frac{1248}{2262 \tau^{2}}\right) v_{n}(t) \\
+ & \left(\frac{1248}{2262 \tau}\right)^{2} \int_{0}^{t} e^{-\frac{1248}{2262 \tau}(t-s)}\left(\dot{v}_{n}(s)+\frac{1}{\tau} v_{n}(s)\right) d s=f_{n}(t) .
\end{aligned}
$$

Letting

$$
w_{n}(t)=\left(\frac{1248}{2262 \tau}\right)^{2} \int_{0}^{t} e^{-\frac{1248}{2262 \tau}(t-s)}\left(\dot{v}_{n}(s)+\frac{1}{\tau} v_{n}(s)\right) d s
$$

we obtain,

$$
\dot{w}_{n}(t)=\left(\frac{1248}{2262 \tau}\right)^{2}\left(\dot{v}_{n}(t)+\frac{1}{\tau} v_{n}(t)\right)-\frac{1248}{2262 \tau} w_{n}(t) .
$$

We write (41) as first order vector systems by defining new variables

$$
\begin{aligned}
u_{1}^{n}(t) & =v_{n}(t) \\
u_{2}^{n}(t) & =\dot{v}_{n}(t) \\
u_{3}^{n}(t) & =w_{n}(t) \\
d & =\frac{1248}{2262 \tau} \\
\Delta_{n} & =\frac{2262}{\rho} \lambda_{n}-\frac{1248}{2262 \tau^{2}} .
\end{aligned}
$$

Then, from (41) we have the equivalent systems

$$
\frac{d}{d t}\left(\begin{array}{l}
u_{1}^{n} \\
u_{2}^{n} \\
u_{3}^{n}
\end{array}\right)=\left(\begin{array}{ccc}
0 & 1 & 0 \\
-\Delta_{n} & \frac{-1014}{2262 \tau} & -1 \\
\frac{d^{2}}{\tau} & d^{2} & -d
\end{array}\right)\left(\begin{array}{l}
u_{1}^{n} \\
u_{2}^{n} \\
u_{3}^{n}
\end{array}\right)+\left(\begin{array}{c}
0 \\
f_{n} \\
0
\end{array}\right) .
$$


Letting

$$
A_{n}=\left(\begin{array}{ccc}
0 & 1 & 0 \\
-\Delta_{n} & \frac{-1014}{2262 \tau} & -1 \\
\frac{d^{2}}{\tau} & d^{2} & -d
\end{array}\right)
$$

we find

$$
\begin{aligned}
\left|\zeta I-A_{n}\right|= & \zeta^{3}+\left(\frac{1014}{2262 \tau}+d\right) \zeta^{2}+\left(\frac{1014}{2262 \tau} d+d^{2}+\Delta_{n}\right) \zeta \\
& +\Delta_{n} d+\frac{d^{2}}{\tau} .
\end{aligned}
$$

The Routh-Hurwitz Theorem [G] tells us that the roots of the polynomial equation

$$
z^{3}+a_{1} z^{2}+a_{2} z+a_{3}=0
$$

have negative real parts if the following conditions are met

(i) $a_{1}>0$

(ii) $\left|\begin{array}{cc}a_{1} & a_{3} \\ 1 & a_{2}\end{array}\right|>0$

(iii) $a_{3}>0$.

Thus, the roots of $\left|\zeta I-A_{n}\right|=0$ have negative real parts if

$$
\left|\begin{array}{cc}
\frac{1014}{2262 \tau}+d & \Delta_{n} d+\frac{d^{2}}{\tau} \\
1 & \frac{1014}{2262 \tau} d+d^{2}+\Delta_{n}
\end{array}\right|>0 .
$$

Thus, the eigenvalues of $A_{n}$ have negative real parts if

$$
d\left(\frac{1014}{2262 \tau}+d\right)^{2}+\frac{1014}{2262 \tau} \Delta_{n}-\frac{d^{2}}{\tau}>0
$$

Now, recalling that

$$
\begin{aligned}
d & =\frac{1248}{2262 \tau} \\
\Delta_{n} & =\frac{2262 \lambda_{n}}{\rho}-\frac{1248}{2262 \tau^{2}},
\end{aligned}
$$


we find that simple calculations reveal that inequality (49) is equivalent to

$$
\lambda_{n}>0
$$

Since condition (50) holds for the eigenvalues of $(38),(39)$, we see that the eigenvalues of $A_{n}$ have negative real part and hence, the zero solution of (36) with $f=0$ is asymptotically stable. That is, all modes of (36) are exponentially damped.

From (42), (44), (45), and (46) we have that the temporal coefficients of (40) satisfy

$$
\left(\begin{array}{c}
v_{n}(t) \\
\dot{v}_{n}(t) \\
w_{n}(t)
\end{array}\right)=e^{t A_{n}}\left(\begin{array}{c}
v_{n}(0) \\
\dot{v}_{n}(0) \\
0
\end{array}\right)+\int_{0}^{t} e^{(t-s) A_{n}}\left(\begin{array}{c}
0 \\
f_{n}(s) \\
0
\end{array}\right) d s
$$

where for each $n$,

$$
e^{t A_{n}}\left(\begin{array}{c}
v_{n}(0) \\
\dot{v}_{n}(0) \\
0
\end{array}\right) \longrightarrow\left(\begin{array}{l}
0 \\
0 \\
0
\end{array}\right), \quad t \rightarrow \infty
$$

\section{$6 \quad$ Vibration of a Rubber Rod with a Tip Mass}

In this section we consider the Lagrange formulation for longitudinal vibrations of a rubber rod fixed at $x=0$ with a tip mass at $x=b$. For this we return to the linearized equation (34), set $q=0$ and adjoin the boundary condition

$$
M \frac{\partial^{2} u_{c}}{\partial t^{2}}(t, b)=-\left.S\right|_{x=b}+F(t)+M g,
$$

where $M$ is the tip mass, $g$ the gravitational constant, $F$ is the force applied to the tip mass, and $S=A_{c} \sigma_{L}$ is the internal (engineering) linearized stress resultant given by, in this case,

$$
S=2262 A_{c} \partial_{x} u_{c}-\frac{1014 A_{c}}{\tau} \int_{0}^{t} \partial_{x} u_{c}(s, x) e^{-(t-s) / \tau} d s .
$$

Using (35) we obtain

$$
S=\rho A_{c} \partial_{x} v,
$$


and from (34)

$$
\partial_{t}^{2} u_{c}=v_{x x}+\frac{1}{\rho A_{c}} q .
$$

For the problem of the vibration of a rod with a tip mass, as indicated in the first paragraph above, we have $q=0$. Thus from (55) we have

$$
\partial_{t}^{2} u_{c}=v_{x x}
$$

Moreover from (52), (54) and (56) we have

$$
M v_{x x}(t, b)=-\rho A_{c} v_{x}(t, b)+F(t)+M g .
$$

Formally employing the Sturm-Liouville theory and series solution from the previous section in (57), we have

$$
\sum_{n}\left[-M \lambda_{n} \varphi_{n}(b)-\rho A_{c} \frac{1}{h_{1}} \varphi_{n}(b)\right] v_{n}(t)=F(t)+M g
$$

We may then formally write

$$
F(t)+M g=\sum_{n=1}^{\infty}\left\langle F(t)+M g, \varphi_{n}\right\rangle \varphi_{n}
$$

where $\langle$,$\rangle is the L_{2}$-inner-product. Then, using (58) and (59), and the fact that, under appropriate regularity assumptions, the series in (59) converges pointwise for every $x$ in $(0, b)$, we have

$$
\left(-M \lambda_{n}-\frac{1}{h_{1}} \rho A_{c}\right) v_{n}(t)=\left\langle F(t)+M g, \varphi_{n}\right\rangle
$$

or

$$
v_{n}(t)=\frac{\left\langle F(t)+M g, \varphi_{n}\right\rangle}{-M \lambda_{n}-\frac{1}{h_{1}} \rho A_{c}}
$$

Thus,

$$
v(t, x)=\sum_{n=1}^{\infty} \frac{\left\langle F(t)+M g, \varphi_{n}\right\rangle}{-M \lambda_{n}-\frac{1}{h_{1}} \rho A_{c}} \varphi_{n}(x) .
$$

We have used formal arguments with (57) and (58), enabling us to write (61) for $v_{n}(t)$, and thus (62) for $v(t, x)$. However, having this form for $v(t, x)$, 
we can invert our arguments to give a rigorous development for this representation (62). Specifically, using known facts about completeness of the eigenfunctions of Sturm-Liouville problems and convergence properties of expansions in terms of thes eigenfunctions we can establish (62) with complete rigor. Indeed, taking $a=0, \quad h_{0}=0$ in (38), we can readily compute the eigenvalues and eigenfunctions and validate our formal steps.

Returning to (35) we can solve for $u_{c}$ giving the displacement of the rubber rod. Thus,

$$
\begin{aligned}
u_{c}(t, x)= & \frac{\rho}{2262} \int_{0}^{t} e^{-\frac{1248}{2262}(t-s)}\left(v_{s}+\frac{1}{\tau} v\right) d s+e^{-\frac{1248}{2262 \tau} t} u_{c}(0, x) \\
= & \frac{\rho}{2262} e^{-\frac{1248}{2262 \tau} t} v(0, x)+\frac{1014}{2262} \frac{\rho}{2262 \tau} \int_{0}^{t} e^{-\frac{1248}{2262 \tau}(t-s)} v(s, x) d s \\
& +e^{\frac{-1248}{2262 \tau} t} u_{c}(0, x) .
\end{aligned}
$$

Thus, using (62) we have

$$
\begin{aligned}
u_{c}(t, x) & =\frac{\rho}{2262} e^{-\frac{1248}{2262 \tau} t} \sum_{n=1}^{\infty} \frac{\left\langle F(0)+M g, \varphi_{n}\right\rangle}{M \lambda_{n}-\frac{1}{h_{1}} \rho A_{c}} \varphi_{n}(x) \\
& +\frac{1014}{2262} \frac{\rho}{2262 \tau} \int_{0}^{t} e^{-\frac{1248}{2262 \tau}(t-s)} \sum_{n=1}^{\infty} \frac{\left\langle F(s)+M g, \varphi_{n}\right\rangle}{-M \lambda_{n}-\frac{1}{h_{1}} \rho A_{c}} \varphi_{n}(x) d s \\
& +e^{-\frac{1248}{2262 \tau} t} u_{c}(0, x) .
\end{aligned}
$$

We see that (63) is an explicit formula for the displacement of the rubber rod. If

$$
|F(s)| e^{\frac{1248}{2262 \tau} s} \leq C e^{-\epsilon s}, \epsilon>0
$$

we see from (63) that, asymptotically, only the weight of the tip mass determines the displacement of the rod.

\section{Conclusion}

We have presented a dynamic simulation of rubber viscoelasticity based on reptation models. Deformed molecular chains or segments entrapped between cross-linked molecules or molecular chains tend to return to their positions prior to deformation. This is due to the fact that their original positions 
are positions of lower energy and higher entropy. However, due to the physical and electrostatic barriers created as a result of new configurations and conformations the entrapped and strained molecules only creep to their original confirmation. The model presented adheres to these observations and also points out the interaction of density and relaxation time on the creep behaviour. These models can be related in a direct manner to previously derived (pseudo-phenomenological) models based on data from quasi-static and dynamic experiments with rubber rods in uniaxial tension and in shear.

\section{References}

[ABP] A. Ackleh, H.T. Banks, and G.A. Pinter, Well-Posed Results for Models of Elastomers, Tech. Rep. CRSC-TR00-21, NCSU, Sept. 2000; J. Math. Analysis Appl., submitted.

[BPP] H.T. Banks, G.A. Pinter and L.K. Potter, "Existence of Unique Weak Solutions to a Dynamical System for Nonlinear Elastomers with Hysteresis", CRSC-TR98-43, NCSU, Nov. 1998; Differential and Integral Equations, 13 (2000), pp. 1001-1024.

[BPPGY] H.T. Banks, G.A. Pinter, L.K. Potter, M.J. Gaitens and L.C. Yanyo, "Modeling of Nonlinear Hysteresis in Elastomers Under Uniaxial Tension", CRSC-TR99-09, NCSU, Feb. 1999; J. Intelligent Material Systems and Structures 10 (1999), pp. 116-134.

[CL] E. Coddington and N. Levinson, Theory of Ordinary Differential Equations, McGraw-Hill, New York 1955.

[DE] M.Doi and S.F. Edwards, The Theory of Polymer Dynamics, Oxford, New York, 1986.

[G] F.R. Gantmacher, Matrix Theory, Vol. 2, Chelsea Publishing Company, New York 1959.

$[\mathrm{J}] \quad$ A.R. Johnson, Modeling and Viscoelastic Materials Using Internal Variables, The Shock and Vibration Digest 31 (1999), pp.91-100. 
[JQYD] A.R. Johnson, C.J. Quigley, D.G. Young and J.A. Danik, Viscohiperelastic Modeling of Rubber Vulcanization, Tire Sci. Technol. 21(1993), pp. 179-199.

[JS] A.R. Johnson and R.G. Stacer, Rubber Viscoelasticity Using the Physically Constrained System's Stretches as Internal Variables, Rubber Chemistry and Technology, 66 (1993) pp. 567-577.

[O] R.W. Ogden, Non-Linear Elastic Deformations, Ellis Horwood Limited, Chichester, 1984.

[S] L.H. Sperling, Introduction to Physical Polymer Science, John Wiley \& Sons, 1992.

[T] L.R.G. Treloar, The Physics of Rubber Elasticity, Clarendon, Oxford 1975 .

[VL] K.C. Valanis and R.F. Landel, The Strain-energy Function of a Hyperelastic Material in Terms of the Extension Ratios, J. Applied Physics, 38(1967), pp.2997-3002. 